\title{
Australian Neonatal Nurses' Perceptions of Neurodevelopmental Care
}

\author{
Griffiths Na, Galea Ca,b, Psaila Ka,c \\ a Grace Centre for Newborn Care, The Sydney Children's Hospital Network, Westmead, Australia \\ ${ }^{\mathrm{b}}$ Cerebral Palsy Alliance, Allambie, Australia \\ c Western Sydney University, Parramatta, Australia
}

\section{Aims/Purpose}

The value of supporting neurodevelopmental outcomes by reducing stress and noxious stimuli in the NICU has been established in the literature over the past 20 years. ${ }^{1,2}$ Developmental care and its application in the clinical setting is reported as inconsistent, yet there is no literature exploring neonatal nurses' perceptions of developmental care and its application within the Australian context. We undertook this survey to gauge the current practices across Australia as part of a planning exercise for future neurodevelopmental care (NDC) training and implementation of NIDCAP.

\section{Methods}

The survey was modified from a tool exploring Neonatal Nurses Perceptions of Family Centered Care and Developmental Practices in the United States of America. ${ }^{3}$ The modified survey consisted of thirty six questions exploring nurse's personal perceptions and beliefs relating to family centered care, developmental care and skin to skin practices. Additional questions relating to NDC education attendance were included by local researchers. The survey was distributed via the online Qualtrics ${ }^{\odot}$ platform to seven hundred and eighty three $(\mathrm{n}=783)$ specialty neonatal nurses in Australia.

\section{Results}

One-hundred and seventy three ( $\mathrm{n}=173)$ nurses completed the survey with a 22 percent response rate. Statistical analysis of the data utilised Fishers Exact test for association and unadjusted odds ratios. Statistically significant associations were demonstrated for: Place of employment (nursery versus combined neonatal intensive care/nursery unit) with combined units associated with increased support for open visiting hours $(\mathrm{p}=0.023)$ and skin to skin holding $(\mathrm{p}=0.009)$. Supportive positioning $(\mathrm{p}=0.026)$ and recognition of the influence of the NICU layout/design $(\mathrm{p}=0.055)$ was also positively associated with combined units. Respondent Post Graduate education levels were associated with increased recognition of the influence of the NICU environment $(\mathrm{p}=0.025)$. Whilst lower levels of agreement (not statistically significant) were seen in all groups relating to parental involvement in care, and support of peers or the multidisciplinary team to facilitate skin to skin holding.

Differences were noted between rural $(\mathrm{n}=21)$ and metropoli$\tan (n=150)$ respondents access to NDC education. Seventy one (71) percent of NDC training for rural respondents occurred outside their hospital. With rural center respondents two times more likely to have completed education greater than six months

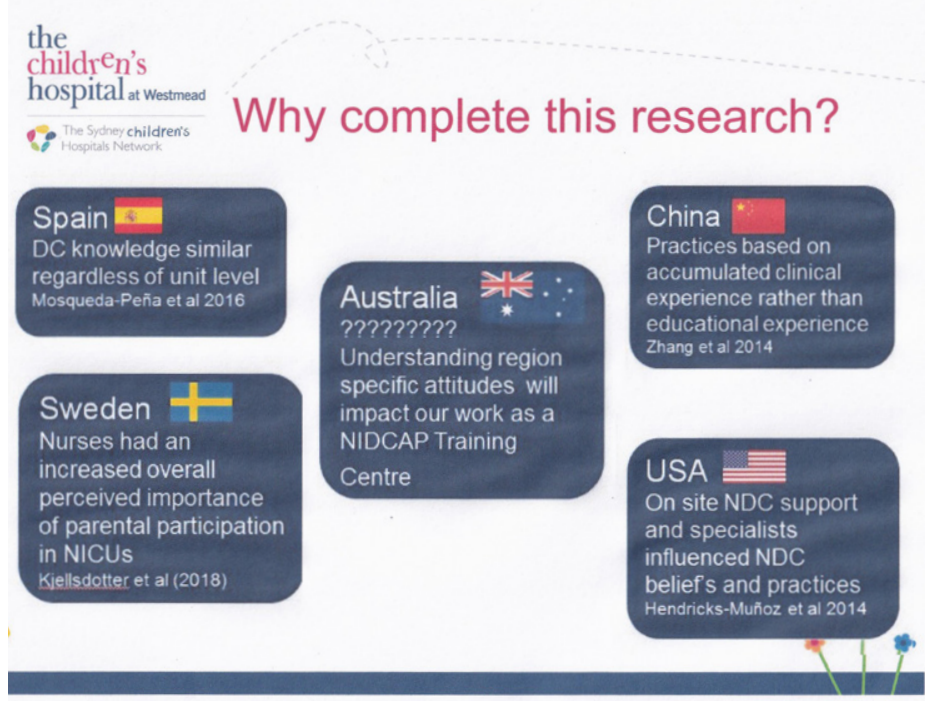

ago $(\mathrm{p}=0.005)$. Of concern the majority of respondents $(64 \%)$ who had attended education in the past two years had received less than one hour of NDC education. With eight percent indicating they had never attended NDC education.

\section{Conclusion}

Respondents in this study demonstrated high levels of support for the concept of NDC. In the context of this survey, location, place of employment and level of education were identified as influencing the application of NDC components. Given the NIDCAP community's role in providing educational and consultative support in the NICU and special care nursery settings to ensure effective delivery of neurodevelopmentally supportive, individualized, and family-centered care understanding the potential influence of location and country specific factors is essential. Exploring the unique geographical differences seen within the global healthcare setting can ensure context specific needs are met when implementing NDC education programs including NIDCAP.

\footnotetext{
References:

1. D'Agata AL, Sanders MR, Grasso DJ, Young EE, Cong X, McGrath JM. Unpacking the burden of care for infants in the NICU. Infant Mental Health Journal, 2017; 38:306-17.

2. Spence K. Historical trends in neonatal nursing: Developmental care and NIDCAP. The Journal of Perinatal and Neonatal Nursing 2016;30:3:273-76.

3. Hendricks-Muñoz KD, Louie M, Li Y, Chhun N, Prendergast CC, Ankola P. Factors that influence neonatal nursing perceptions of family-centered care and developmental care practices. American Journal of Perinatology 2010;27:3: 193-200.
} 